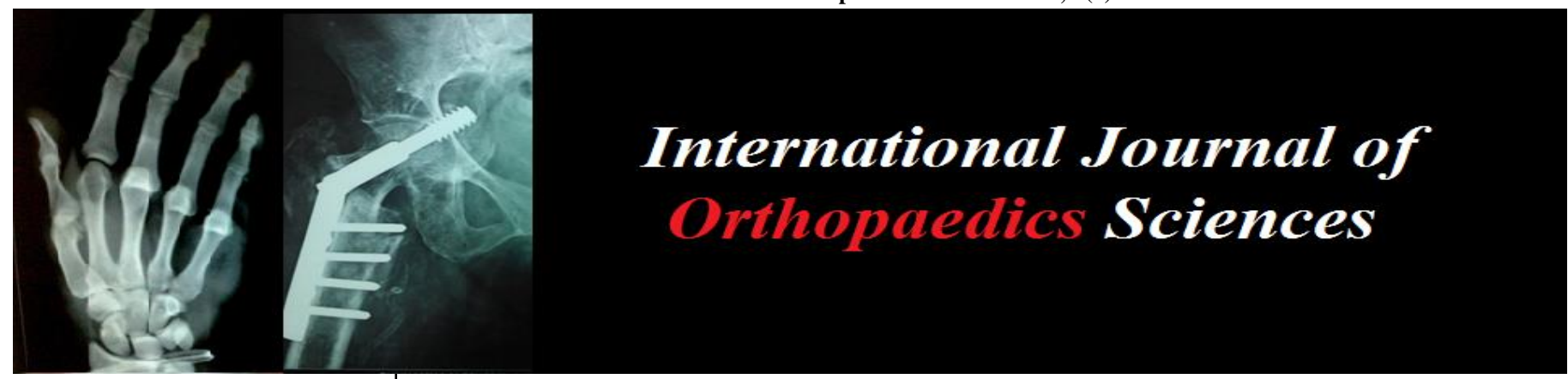

ISSN: $2395-1958$

IJOS 2019; 5(3): 275-277

(C) 2019 IJOS

www.orthopaper.com

Received: 09-05-2019

Accepted: 13-06-2019

Ruchit Khera

Assistant Professor, Department of Orthopaedics, Swami Rama

Himalayan University,

Himalayan Institute of Medical

Sciences, Swami Ram Nagar

Jolly Grant, Dehradun,

Uttarakhand, India

Atul Agrawal

Professor, Department of

Orthopaedics, Swami Rama

Himalayan University,

Himalayan Institute of Medical

Sciences, Swami Ram Nagar,

Jolly Grant, Dehradun,

Uttarakhand, India
Correspondence

Efstathios Drampalos

Assistant Professor, Department of Orthopaedics, Swami Rama

Himalayan University,

Himalayan Institute of Medical

Sciences, Swami Ram Nagar,

Jolly Grant, Dehradun,

Uttarakhand, India

\section{Conjoint bicondylar hoffas fracture with ipsilateral femur and tibia shaft fracture in pediatric patient}

\section{Ruchit Khera and Atul Agrawal}

DOI: https://doi.org/10.22271/ortho.2019.v5.i3e.1539

\section{Abstract}

Hoffa's Fracture i.e. intra-articular coronal sheer fractures of femoral condyle is a rare presentation in adults, and much more rarer in children. We wish to bring up a case of 05 year child with closed conjoint bicondylar hoffa's fracture with ipsilateral open grade III A shaft femur fracture with ipsilateral closed tibia fracture. Till date case of conjoint hoffa's fracture with floating knee have not been reported in literature. Patient have been successfully treated with open reduction and internal fixation with good functional outcome.

Keywords: Conjoint, hoffa's, floating knee, pediatric fractures

\section{Introduction}

Fracture of femur is a common presentation in patient with high velocity trauma, however fracture of femoral condyles in coronal plane, first of all described by Albert Hoffa in 1904 [1]. It is an uncommon injury in adults and more rarer in pediatric population. Diagnosing these need very high index of suspicion and are usually missed on conventional radiographs. Conjoint bicondylar pattern of fracture is rarely described in literature, specially in pediatric population. As per current literature analysis using pubmed search engine only 9 cases of Hoffa's fractures have so far been reported in pediatric population with only two cases with conjoint hoffa's fracture ${ }^{[2,3]}$. Pubmed search was done using keywords; Hoffa, Hoffa's, fracture, pediatric, children. We hereby report a case of 05 year old child with conjoint bicondylar Hoffa's fracture with ipsilateral femur shaft and tibia fracture, successfully managed with open reduction and internal fixation. As per our knowledge none of the case of conjoint Hoffa's fracture with floating knee being reported till now.

\section{Case report}

Concent was obtained for publication of case report from legal guardians of patient and approval of institutional ethical committee was obtained for the same.

A 10 year old male child presented in emergency department 7 hours after road traffic accident due to which he sustained injury to his right lower limb. Patient had grossly contaminated $10 \times 7 \mathrm{~cm}$ open wound over anteromedial aspect of upper third thigh with visible bone and artery with swelling around ipsilateral knee and leg, with knee held in flexion with resistance to passive extension of the same. On examination patient had open visible fracture femur with abnormal mobility, crepitus in upper third of tibia with restricted and painful movements around knee without any vascular or neurological deficit. Patient was evaluated radiologically with xrays of knee, hip with thigh and knee with leg with ankle-anteroposterior and lateral views, and was found to have coronal plane fracture of femoral condyles with fracture shaft of femur with ipsilateral tibia fracture. Patient was initially taken for surgery where debridement of compound wound over femur and stabilisation with external fixator for femur was done along with stabilisation with posterior POP slab for tibia and distal femur fracture.

Patient was kept under observation in view of any signs of infection at compounding site, before attempting any internal fixation for other fractures. Patient was taken up for elective surgery for fixation of femoral condyle fracture and tibia fracture after 7 days. Femoral condyle was approached with standard anterior incision with medial parapatellar approach 
dislocating patella laterally and was found to have bicondylar coronal split of femoral condyles. Fractures were reduced with $\mathrm{k}$ wire (Joy stick method under direct vision) and fixed with three cannulated screws (Extra-physeal) along with close reduction and fixation of tibia using ender's nail.

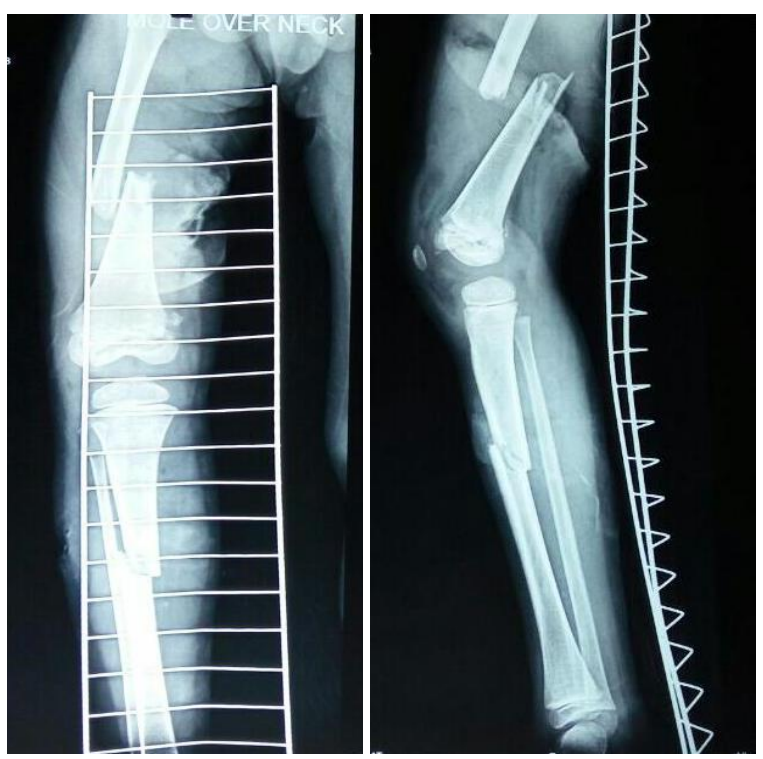

Fig 1: Pre-operative Xrays

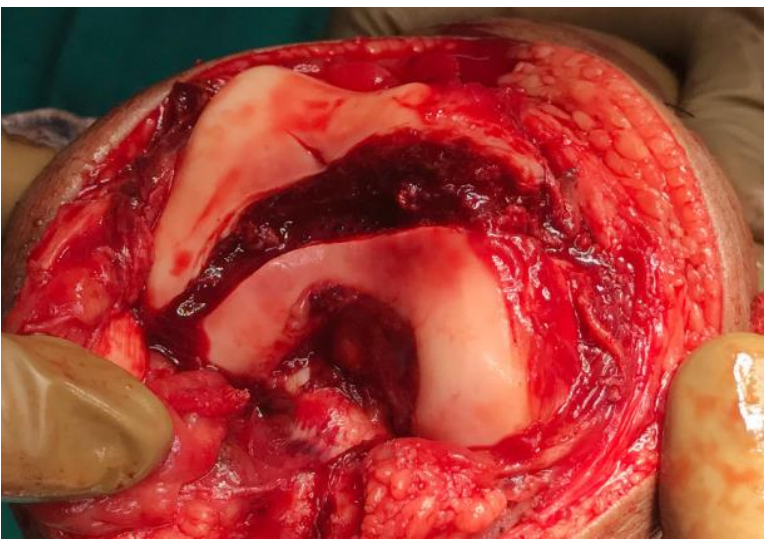

Fig 2: Intra-operative Pictures showing conjoint bicondylar Hoffa fracture

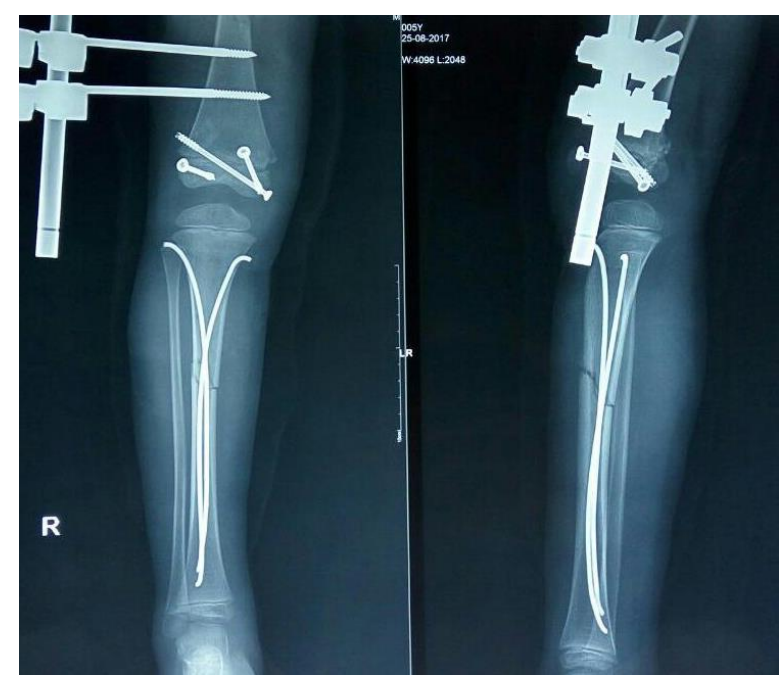

Fig 3: Post-operative xrays showing Fixation of conjoint bi condylar Hoffa fracture using cannulated cancellous screws.

Post operatively patient was kept on below knee plaster for 2 weeks after which knee mobilisation was started. Weight bearing was delayed in view of fracture of femur and tibia. Knee Range of motion gained at 12 weeks post operatively was 0-130 degrees actively.

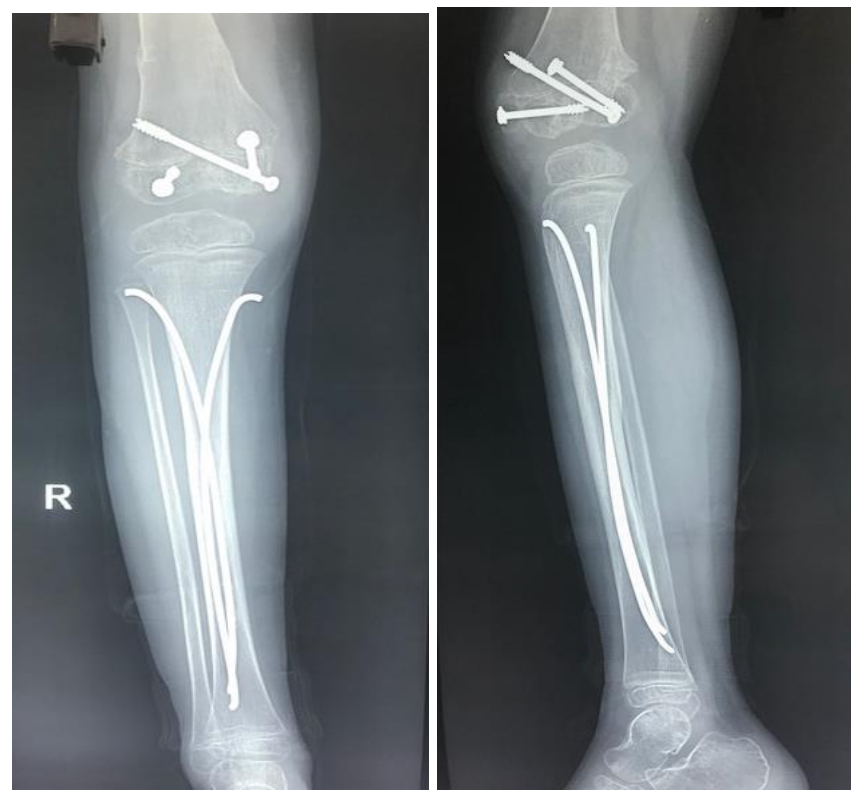

Fig 4: Xrays at union 3 months

\section{Discussion}

Hoffa's fracture refers to coronal split fracture of femoral condyles with intra-articular extension. It can be lateral hoffa's fracture or medial hoffa's fracture. Lateral condyle hoffas are more frequently found than medial condyle fractures, believed to be due to physiological genu valgum. ${ }^{[3]}$ Isolated medial hoffa's fracture was first described by Bali et $a l$. in ${ }^{[4]}$. These usually result from high velocity trauma.

Hoffas fracture is classified as described by Letenneur et al. ${ }^{[5]}$ as:

Type I Vertical fracture involving the entire condyle parallel to posterior cortex of femur.

Type II Fractures of variable sizes horizontal to base of the condyle.

Type III Fracture oblique to the femur. Author reports worst results with Type III injuries.

Conjoint hoffas fracture has not been classified in any classification system. This is a first report of conjoint hoffas fracture with ipsilateral femur and tibia fracture (Floating knee) in pediatric population. Plain radiograph and computed tomography helps in diagnosis and surgical planning for management of fracture. Diagnosis with plain radiographs need high index of suspicion, especially in minimally displaced fractures where they may be missed or misinterpreted as unicondylar Hoffa fracture.

Surgical stabilization of fracture is considered to be ideal treatment for hoffas fracture considering functional outcomes. Reduction in such fractures is belived to be difficult due to lack of soft tissue attachments, which also make them prone to avascular necrosis and non unions. McDonough et al. reported a case of non union of hoffas fracture in child. ${ }^{[6]}$

Reduction of isolated lateral condyle hoffas fracture can be done using lateral approach, however in conjoint bicondylar Hoffa anterior mid line incision with medial parapatellar approach with lateral dislocation of patella provides accurate reduction and direct visualization of articular surface. Literature has evidence of arthroscopic assisted reduction and management of hoffas fracture leading to less soft tissue 
dissection and blood loss however that is restricted to fresh injuries ${ }^{[3]}$.

Associated Ipsilateral femur and tibia shaft fractures make such injuries more complicated, especially in regard to delay in definitive fixation in case of compound injuries, alignment of limb, early mobilization of knee, and functional outcomes. In our case patient had Open Grade III A fracture shaft femur, leading to delay in internal fixation. However, Patient had ROM of 0-90 degree at 6 wks follow-up and 0-130 degree at 12 wks. Union at femur shaft, condyles of femur and tibia were achieved at 12 wks follow up and implant removal was done at 16 wks.

We conclude that in conjoint Hoffa fracture should be kept in mind in children also and best modality of treatment of such fractures is with internal fixation to prevent best functional outcomes in these patients.

\section{References}

1. Hoffa A. Lehrbuch der Frakturen und Luxationen. Stuttgart: Verlag von Ferdinand Enke. 1904, 451.

2. Harna B, Goel A, Singh P, Sabat D. Pediatric conjoint Hoffa's fracture: An uncommon injury and review of literature. J Clin Orthop Trauma. 2017; 8(4):353-354.

3. Lal H, Bansal P, Khare R, Mittal D. Conjoint bicondylar Hoffa fracture in a child: a rare variant treated by minimally invasive approach. J Orthop Traumatol. 2011; 12(2):111-4.

4. Bali K, Mootha AK, Prabhakar S. Isolated Hoffa fracture of the medial femoral condyle in a skeletally immature patient. Bull. NYU Hosp. Jt. Dis. 2011; 69:335-338.

5. Letenneur J, Labour PE, Rogez IM. Fractures de Hoffa: a propos de 20 observations. Ann. Chir. 1978; 32:213-219.

6. PW McDonough, Bernstein RM. Nonunion of a Hoffa fracture in a child. J. Orthop. Trauma. 2000; 14:519-521. 